\title{
Parallel and Serial Sensory Processing in Developing Primary Somatosensory and Motor Cortex
}

\author{
${ }^{\circledR}$ Lex J. Gómez, ${ }^{1,2,5}{ }^{\oplus}$ James C. Dooley, ${ }^{2,5}{ }^{\circledR}$ Greta Sokoloff, ${ }^{2,5}$ and ${ }^{\circledR}$ Mark S. Blumberg ${ }^{1,2,3,4,5}$ \\ ${ }^{1}$ Interdisciplinary Graduate Program in Neuroscience, University of Iowa, Iowa City, Iowa 52242, ${ }^{2}$ Department of Psychological and Brain Sciences, \\ University of Iowa, Iowa City, Iowa 52242, ${ }^{3}$ Department of Biology, University of Iowa, Iowa City, Iowa 52242, ${ }^{4}$ Iowa Neuroscience Institute, \\ University of Iowa, Iowa City, Iowa 52242, and ${ }^{5}$ DeLTA Center, University of Iowa, Iowa City, Iowa 52242
}

It is generally supposed that primary motor cortex (M1) receives somatosensory input predominantly via primary somatosensory cortex (S1). However, a growing body of evidence indicates that M1 also receives direct sensory input from the thalamus, independent of S1; such direct input is particularly evident at early ages before M1 contributes to motor control. Here, recording extracellularly from the forelimb regions of S1 and M1 in unanesthetized rats at postnatal day (P) 8 and P12, we compared S1 and M1 responses to self-generated (i.e., reafferent) forelimb movements during active sleep and wake, and to other-generated (i.e., exafferent) forelimb movements. At both ages, reafferent responses were processed in parallel by $S 1$ and M1; in contrast, exafferent responses were processed in parallel at P8 but serially, from S1 to M1, at P12. To further assess this developmental difference in processing, we compared exafferent responses to proprioceptive and tactile stimulation. At both P8 and P12, proprioceptive stimulation evoked parallel responses in S1 and M1, whereas tactile stimulation evoked parallel responses at P8 and serial responses at P12. Independent of the submodality of exafferent stimulation, pairs of S1-M1 units exhibited greater coactivation during active sleep than wake. These results indicate that S1 and M1 independently develop somatotopy before establishing the interactive relationship that typifies their functionality in adults.

Key words: development; motor cortex; movement; sensory; sleep; somatosensory cortex

Significance Statement

Learning any new motor task depends on the ability to use sensory information to update motor outflow. Thus, to understand motor learning, we must also understand how animals process sensory input. Primary somatosensory cortex (S1) and primary motor cortex (M1) are two interdependent structures that process sensory input throughout life. In adults, the functional relationship between S1 and M1 is well established; however, little is known about how S1 and M1 begin to transmit or process sensory information in early life. In this study, we investigate the early development of S1 and M1 as a sensory processing unit. Our findings provide new insights into the fundamental principles of sensory processing and the development of functional connectivity between these important sensorimotor structures.

\section{Introduction}

Motor learning, including the ability to adapt motor output in a contextually relevant manner, depends on the processing and integration of sensory input within sensory and motor structures (Pavlides et al., 1993; Rosenkranz and Rothwell, 2012; Mathis et al., 2017). Primary somatosensory cortex (S1) and primary motor cortex (M1) are two structures that exemplify this interplay between sensory and motor modalities. According to the

\footnotetext{
Received 0ct. 8, 2020; revised Dec. 23, 2020; accepted Feb. 16, 2021.

Author contributions: L.J.G., J.C.D., G.S., and M.S.B. designed research; L.J.G. performed research; L.J.G. and

J.C.D. analyzed data; L.J.G., J.C.D., and M.S.B. wrote the paper.

This work was supported by the National Institutes of Health Grant R37-HD081168 (to M.S.B.).

The authors declare no competing financial interests.

Correspondence should be addressed to Mark S. Blumberg at mark-blumberg@uiowa.edu.

https://doi.org/10.1523/JNEUROSCI.2614-20.2021

Copyright $\odot 2021$ the authors
}

classic model, S1 and M1 form a sensorimotor loop wherein sensory input first arrives in S1 before it is conveyed to M1 to modulate motor outflow (Vidoni et al., 2010; Zagha et al., 2013; Umeda et al., 2019). Ample evidence, mostly in adult animals, supports this model. For example, S1 sends excitatory axonal projections to M1 (Hooks et al., 2011; Mao et al., 2011; RoccoDonovan et al., 2011), ablating S1 reduces or abolishes activity in M1 (Goldring et al., 1970; Farkas et al., 1999), silencing S1 impairs motor adaptation (Sakamoto et al., 1989; Mathis et al., 2017), and the latency of evoked and spontaneous sensory responses is typically shorter in S1 than M1 (Ferezou et al., 2007; Chakrabarti et al., 2008; McVea et al., 2012; An et al., 2014). However, the existence of short-latency sensory responses in M1 (Asanuma et al., 1979; Horne and Tracey, 1979; Lemon and van der Burg, 1979; Tracey et al., 1980; Herman et al., 1985; Asanuma and Mackel, 1989) and evidence of S1's role in motor control (Sasaki and Gemba, 1984; Matyas et al., 2010; Halley et 
al., 2020) suggest that the conventional division of S1 and M1 into distinct sensory and motor areas, respectively, is questionable (Hatsopoulos and Suminski, 2011; Ebbesen et al., 2018).

In early development, the issue of how best to categorize the sensory and motor functions of S1 and M1 is especially apposite. M1 does not assume its motor functionality until relatively late in development (Chakrabarty and Martin, 2000; Martin, 2005; Young et al., 2012). In rats, motor outflow from M1 is not detectable without pharmacological manipulation until postnatal day (P)25 (Young et al., 2012; Singleton et al., 2021); before this age, M1, like S1, appears to function exclusively as a sensory structure (Tiriac et al., 2014; Dooley and Blumberg, 2018). Also, the initial development of a sensory map in motor cortex is not exclusive to rats (Chakrabarty and Martin, 2005). Thus, in adults, it may be that M1's motor functions obfuscate its sensory functions, and so the absence of motor outflow at early ages helps to reveal M1's sensory architecture. In general, developmental analyses of sensory processing can effectively reveal the foundational functional properties on which adult operations are built.

To fully and accurately decipher the functional interactions between S1 and M1, it is also important to consider the influence of behavior on sensory processing. For example, when P8 and P12 rats exhibit self-generated forelimb movements, sensory feedback from those movements (i.e., reafference) is conveyed from the thalamus in parallel to S1 and M1 (Dooley and Blumberg, 2018). It is not known, however, whether such parallel processing is a specific feature of reafference in early development. Indeed, previous studies of S1-M1 sensory processing in adults have focused almost exclusively on sensory signals arising from externally generated stimuli (i.e., exafference). (It should also be noted that the self-generated movements that give rise to reafference are typically accompanied by corollary discharge signals (Crapse and Sommer, 2008), even in rats as early as P8 (Mukherjee et al., 2018)). Here, we compare reafferent and exafferent processing in $\mathrm{S} 1$ and $\mathrm{M} 1$ at $\mathrm{P} 8$ and P12. In addition, we examine the importance of sensory submodality (i.e., proprioceptive vs. tactile) as well as the modulating influence of behavioral state. We find that all sensory input is processed in parallel by $\mathrm{S} 1$ and M1 until approximately P12, at which time serial processing does emerge, but exclusively in response to tactile input.

\section{Materials and Methods}

All experiments were conducted in accordance with the National Institutes of Health Guide for the Care and Use of Laboratory Animals (NIH Publication No. 80-23) and were approved by the Institutional Animal Care and Use Committee of the University of Iowa.

\section{Subjects}

A total of 32 (16 female) Sprague Dawley rats aged P8 (body weight: $19.1 \pm 1.6 \mathrm{~g}$ ) and P12 (body weight: $29.1 \pm 1.6 \mathrm{~g}$ ) were used in this study. Pups were born to dams housed in standard laboratory cages $(48 \times 20 \times$ $26 \mathrm{~cm}$ ). Food and water were available ad libitum, and animals were maintained on a 12/12 h light/dark schedule. All dams were checked daily for pups and the day of birth was considered P0. To ensure healthy and relatively uniform weight across experimental subjects, litters were culled to eight pups by P3. To circumvent statistical problems associated with litter effects, littermates were always assigned to different experimental groups (Holson and Pearce, 1992). All experiments were performed during the lights-on period.

\section{Experimental approach}

Surgical preparation

All pups were prepared for neurophysiological recording using methods similar to those described previously (Tiriac et al., 2014; Blumberg et al.,
2015; Dooley and Blumberg, 2018). Briefly, on the day of testing, a rat pup with a visible milk-band was removed from its home cage and anesthetized with isoflurane (3-5\%; Phoenix Pharmaceuticals). Surgery was performed on a heating pad to keep pups warm. Stainless steel bipolar hook electromyographic (EMG) electrodes (California Fine Wire) were implanted into the nuchal, right forelimb (biceps brachii), left forelimb (biceps brachii), and right hindlimb (extensor digitorum longus) muscles, and a ground wire was secured transcutaneously. We administered an anti-inflammatory analgesic subcutaneously (carprofen, $5 \mathrm{mg} / \mathrm{kg}$; Putney). A portion of scalp was removed to reveal the skull and a topical analgesic was applied (bupivacaine $0.25 \%$; Pfizer); Vetbond (3M) was also applied to the skin around the skull. Next, a stainless-steel head-fix apparatus was secured to the skull with cyanoacrylate adhesive and accelerant (Insta-Set, Bob Smith Industries). The pup was then transferred to a second surgical station where, with continued isoflurane administration, the pup was secured in a stereotaxic apparatus and holes were drilled to allow for subsequent electrode insertion in M1 (all coordinates from bregma; P8: $+1.0 \mathrm{~mm}$ rostrocaudal (RC), $1.8 \mathrm{~mm}$ mediolateral (ML); P12: + $1.0 \mathrm{~mm} \mathrm{RC,} 2.0 \mathrm{~mm} \mathrm{ML}$ ) and S1 (P8: + $0.5 \mathrm{~mm} \mathrm{RC}$, $3.0 \mathrm{~mm} \mathrm{ML;} \mathrm{P12:}+0.5 \mathrm{~mm} \mathrm{RC}, 3.3 \mathrm{~mm} \mathrm{ML}$ ). After surgery, which lasted a total of $25 \mathrm{~min}$, the pup was transferred to the recording chamber where it was placed on a raised platform and the head-fix was secured to a stereotaxic apparatus. The pup was further secured with surgical tape placed around its torso and the platform. The raised platform allowed the pup's limbs to dangle freely. The pup recovered from anesthesia for at least $1 \mathrm{~h}$ until its brain temperature reached $36-37^{\circ} \mathrm{C}$ and normal sleep/wake cycles were confirmed.

\section{Data acquisition}

Neurophysiological and EMG recordings were collected using a data acquisition system (Tucker-Davis Technologies). To record neural activity, two silicon iridium electrodes, each with 16 sites distributed linearly at $100-\mu \mathrm{m}$ intervals (Model A1x16-5 mm-100-177-A16, NeuroNexus), were lowered into the forelimb regions of S1 and M1 to a depth of $\sim 0.9-1.4 \mathrm{~mm}$. To enable histologic confirmation of electrode location, electrodes were coated before insertion with fluorescent DiI (Life Technologies). An Ag/AgCl ground/reference electrode (Medwire, 0.25 $\mathrm{mm}$ in diameter) was inserted in occipital cortex. Neural activity and EMG data were sampled at 25 and $1 \mathrm{kHz}$, respectively, and were filtered through a digital preamplifier. A video camera (80-100 frames/s; FLIR Integrated Systems) was used to record synchronized behavioral data for subsequent analysis of limb movements.

\section{Experimental design}

We conducted two experiments to determine how sensory input is processed by $\mathrm{S} 1$ and $\mathrm{M} 1$ at P8 and P12. In the first experiment, we compared reafferent and exafferent signals. Spontaneous twitches and wake movements (i.e., self-generated movements that trigger reafference) were recorded for $30 \mathrm{~min}$, during which time the pup cycled freely through sleep and wake. This 30 -min period was followed by a period of stimulation, which consisted of passive movement of the right forelimb (i.e., exafference) using a small wooden dowel to displace the limb. These stimulations $(n=100)$ were delivered $\sim 2-3$ s apart during sleep and wake, and care was taken to stimulate the limb when it was not already moving. Because the experiment was designed such that the period of limb stimulation always followed the period of sleep-wake cycling (i.e., the two periods were not counterbalanced), we did not make direct statistical comparisons between the two periods.

In the second experiment, we compared processing of proprioceptive and tactile input by delivering two types of stimulation: intramuscular electrical stimulation and cutaneous stimulation. Intramuscular stimulation was delivered via an EMG electrode inserted into the right biceps muscle; electrical current was delivered using an isolated pulse stimulator (AM Systems, Model 2100). Intramuscular stimulations consisted of a single, 20 -ms biphasic pulse delivered at $\sim 8$-s intervals (to prevent muscle fatigue). Before neurophysiological recording began, a starting voltage $(5.0-7.2 \mathrm{~V})$ was determined for each pup; if, during stimulus administration, the evoked forelimb movement diminished, the voltage was slowly increased (never $>0.3 \mathrm{~V}$ in total). We did not observe a 
relationship between voltage intensity and the magnitude of the neural response. The stimulation apparatus produced brief transients $(\sim 1-2 \mathrm{~ms}$ in duration) in the electrophysiological record; the transients were later marked as noise and removed. Cutaneous stimulation was delivered using a fine camel-hair brush, which was briefly applied to the glabrous side of the paw without causing noticeable movement of the forelimb; these stimulations were also delivered $\sim 8 \mathrm{~s}$ apart. We delivered 100 stimulations of each type and the order of the stimulation type was counterbalanced between animals. Intramuscular and cutaneous stimulation trials that co-occurred with self-generated movements were discarded from analysis.

\section{Histology}

At the end of the experiment, the pup was euthanized with 10:1 ketamine/xylazine $(>0.08 \mathrm{mg} / \mathrm{kg}$, i.p.) and perfused transcardially with $1 \mathrm{M}$ PBS followed by $4 \%$ paraformaldehyde (PFA). The brain was extracted from the skull and placed in PFA for at least $24 \mathrm{~h}$, after which it was transferred to phosphate buffered sucrose for at least $48 \mathrm{~h}$ before sectioning. The cortex was sectioned coronally $(80 \mu \mathrm{m})$ using a freezing microtome (Leica Microsystems). Electrode location was confirmed using a fluorescent microscope at 2.5-5× magnification (Leica Microsystems). Tissue was then stained for cytochrome oxidase (CO), which enabled identification of cortical layers in S1 and M1 (Dooley and Blumberg, 2018). Electrode placements were reconstructed using fluorescent and $\mathrm{CO}$-stained images, and the cortical layer of each electrode site was determined. The presence of granular layer 4 in S1 was used to demarcate the S1-M1 border.

\section{Data analysis}

Processing of electrophysiological data

Digital records of neurophysiological and EMG data were imported into MATLAB (The MathWorks; RRID: SCR_001622). Raw neurophysiological data were filtered for unit activity (bandpass filter: $500-5000 \mathrm{~Hz}$ ). Putative unit activity was extracted using Kilosort (Pachitariu et al., 2016) and unit templates were visually evaluated to confirm that they were single units, multiunit activity, or noise using Phy2 (Rossant and Harris, 2013). Preliminary analyses were performed to determine whether response profiles of single unit activity and multiunit activity differed. There were no substantial discrepancies between them, thus, all subsequent analyses were conducted using both single unit and multiunit activity (hereafter "units" or "unit activity").

\section{Behavioral analysis}

Behavior and state were assessed by first visualizing the EMG data. To accomplish this, we imported the neurophysiological and EMG data into Spike2 (Cambridge Electronic Design). We separated files into periods of active sleep and wake and identified the associated self-generated movements (myoclonic twitches and wake movements, respectively) using established methods (Seelke and Blumberg, 2008; Tiriac et al., 2014; Dooley and Blumberg, 2018). Active sleep was defined as periods of nuchal muscle atonia punctuated by sharp spikes in the EMG records, indicative of myoclonic twitching; the presence of limb twitching was corroborated using the video record. Within periods of active sleep, we identified individual right forelimb twitches by first rectifying and smoothing $(0.001 \mathrm{~s})$ the right forelimb EMG. EMG events were categorized as twitches if they exceeded a threshold of $3 \times$ the baseline activity and occurred at least $300 \mathrm{~ms}$ after a preceding twitch. Periods of wake were defined based on the presence of high muscle tone as well as highamplitude limb movements observed in the video record. To identify individual wake movements, wake periods were analyzed for instances in which the forelimb EMG increased $5 \times$ above the baseline for at least $300 \mathrm{~ms}$ and occurred at least $300 \mathrm{~ms}$ after a preceding wake movement (Dooley and Blumberg, 2018). Finally, Spike2 was used to mark the onset of forelimb stimulation using video and EMG records; the behavioral state during each stimulation was also recorded for later analysis.

\section{Analysis of neural data}

All analyses of neural data were performed in MATLAB using customwritten scripts. To determine how S1 and M1 respond to self-generated and other-generated movements, perievent time histograms (PETHs) were constructed for each unit using twitches, wake movements, and stimulus presentations as triggers. Next, we determined the mean baseline firing rate from -500 to $-100 \mathrm{~ms}$ before the triggered event. Finally, $\mathrm{z}$-scored PETHs were calculated by subtracting this baseline from the raw PETH and then dividing this value by the standard deviation of the baseline.

To determine whether units in S1 and M1 were "responsive" to a sensory event, we examined PETHs to determine response windows for each stimulus type. Response windows were defined as periods of time that surrounded peak poststimulus activity. For twitches, wake movements, passive limb movements, and intramuscular stimulation, we used a 150-ms window; for cutaneous stimulation, we used a 400-ms window. Units were considered responsive if the mean firing rate within the window was $2 \times$ greater than the expected baseline level of activity.

We did not always observe a neural response in S1 and M1 for each sensory event, be it a self-generated movement or exafferent stimulation. Thus, we determined the percentage of events that evoked a neural response in S1 and M1. For each event type, we calculated the baseline firing rate (as described above) in addition to the mean postevent firing rate over the response windows (twitches, wake movements, passive limb movements, and intramuscular stimulation: $400-\mathrm{ms}$ postevent window; cutaneous stimulation: $800-\mathrm{ms}$ postevent window). S1 and M1 were considered to respond to an individual sensory event if the mean firing rate within the response window was $1.5 \times$ over baseline. To account for variability in baseline firing rates across events, the percentage of events to which S1 (or M1) responded was adjusted for the percentage of events during which the baseline period before the event rose above the average baseline (i.e., the spontaneous firing rate) using the following equation:

$$
\mathrm{R}_{\mathrm{T}}=\mathrm{R}_{\mathrm{o}}-\mathrm{S}(1-\mathrm{S}),
$$

where $R_{T}$ is the true response rate, $R_{o}$ is the uncorrected response rate (determined using the response window), and $\mathrm{S}$ is the spontaneous firing rate (determined using the baseline period). To calculate the percentage of coactivation of S1 and M1 in response to a sensory event, we determined the number of events for which S1 and M1 both responded to the same event and divided that number by the total number of events.

To determine whether S1 and M1 were more likely to respond at the same time (i.e., were coactivated) during active sleep than during wake, we first separated intramuscular and cutaneous stimulations into two groups based on whether they were delivered during active sleep or wake. We then determined the number of stimulation events in which S1 and M1 were coactivated. Using a contingency table, we calculated the observed and expected coactivation frequencies. Finally, we calculated the percentage difference between observed and expected frequencies for active sleep and wake.

\section{Shift predictor analysis}

Although PETHs reveal how units in S1 and M1 respond to a stimulus, they cannot distinguish between coactivation of units due to (1) both areas responding independently to a stimulus or (2) functional interaction between areas in response to the stimulus (e.g., serial processing of sensory input from S1 to M1). To distinguish between these two possibilities, a shift predictor analysis was performed (Alloway et al., 1993; Chakrabarti et al., 2008). This analysis takes advantage of differences in the temporal relationships between pairs of neurons: neurons whose activity is functionally connected display spiking patterns with temporally precise relationships, whereas neurons that are simply responding to the same stimulus, without being functionally connected to one another, do not. The shift predictor analysis separates these temporally precise relationships from the less precise stimulus-driven activity, thus enabling the detection of neuron-neuron interactions.

First, a joint histogram was constructed of M1 unit activity triggered on S1 unit activity, which was in turn triggered on an event (e.g., a twitch). A second histogram, called the shift predictor, was calculated by reconstructing the M1-S1-event histogram using a train of S1 unit activity triggered on all other event presentations (e.g., triggered on all 
twitches except the twitch that originally triggered S1 activity). Shifting the event presentations in this way eliminates temporally precise relationships between S1 and M1 unit pairs, thereby providing an estimate of the stimulus-driven response. By subtracting the shift predictor from the original joint histogram, we derived the "corrected" histogram that reveals the activity because of interactions between pairs of units in S1 and M1.

The shift predictor analysis was performed using 1-ms bins with a 300-ms window and 150-ms offset. Within an individual animal, corrected histograms were calculated for all possible S1-M1 unit pairs and then averaged. The statistical significance of these within-animal-averaged corrected histograms was determined by constructing $99 \%$ confidence bands, calculated by multiplying the square root of the average shift predictor by 2.576 (Alloway et al., 1993; Chakrabarti et al., 2008). Data from individual pups were analyzed further if the average corrected histogram rose above the $99 \%$ confidence band and displayed a single clear peak.

We then determined which unit pairs did and did not contribute to the average peak by examining the corrected histogram of each individual S1-M1 unit pair. We calculated the standard deviation of the baseline over the first $100 \mathrm{~ms}$ of the corrected histogram window ( -150 to $-50 \mathrm{~ms}$ before the trigger). Then, we calculated the ratio of the maximum firing rate of the corrected histogram to that standard deviation and set an arbitrary threshold to categorize corrected histograms as responsive or non-responsive. We then constructed average corrected histograms for the responsive and non-responsive categories, and visually examined them. In this manner, we passed the data through several thresholds to determine the optimal threshold for categorizing individual corrected histograms into those that contributed to the average peak and those that did not. The median threshold used for separation was seven times the standard deviation of the baseline at P8 (except for tactile stimulation, where the median threshold value was three) and four times the standard deviation at P12. The S1-M1 unit pairs whose peaks rose above the threshold were considered responsive. Finally, we calculated the percentage of responsive pairs.

Responsive pairs were further analyzed to determine their individual peak latencies. We first averaged corrected histograms across unique S1 units (e.g., the corrected histogram of "unit 1" in S1 was averaged for all pairwise combinations with M1 units). We then extracted the peak times of these unique S1-M1 unit pairs and categorized them based on their latencies into one of three categories: (1) a peak shifted to the left of zero $(<-4 \mathrm{~ms})$, indicating that M1 drives activity in S1 (M1-to-S1); (2) a peak centered around zero $( \pm 4 \mathrm{~ms})$, indicating that $S 1$ and $\mathrm{M} 1$ receive input from a common source; and (3) a peak shifted to the right of zero $(>4 \mathrm{~ms})$, indicating that $\mathrm{S} 1$ drives activity in $\mathrm{M} 1$ (S1-to-M1). We used $\pm 4 \mathrm{~ms}$ as the cutoff based on a study of S1-M1 communication in adult rats (Petrof et al., 2015); this cutoff is relatively conservative because axons in the infant neocortex are not well myelinated (Curry and Heim, 1966; Salami et al., 2003; Mengler et al., 2014; Marques-Smith et al., 2016), and thus corticocortical transmission speeds may be slower than those in adults. For each stimulus type, we pooled the peak latencies and calculated outliers using a standard method based on the interquartile range (Tukey, 1977). For each stimulus type (within an age), outliers comprised $0-19.8 \%$ of the data. We verified that removal of outliers did not appreciably shift the median of any of the peak-latency distributions.

\section{Statistical analyses}

We used SPSS (IBM) for Windows and MATLAB for all statistical analyses. For all tests, $\alpha$ value was set to 0.05 , unless otherwise specified; when necessary, we corrected for multiple comparisons using the Bonferroni procedure. A Shapiro-Wilk test was used to determine whether the data were normally distributed. We tested for significance using the following non-parametric tests: the Wilcoxon matched-pairs signed-rank test $(W)$ for paired samples, the Mann-Whitney $(U)$ test for two independent samples, the Kruskal-Wallis test $(H)$ for more than two groups, and the $\chi^{2}$ test for categorical data. Group median data are used for statistical comparisons, unless otherwise specified. Box plots are used to represent the 25th, 50th, and 75th percentiles; minimum and maximum values are represented by whiskers. For $\chi^{2}$ tests, effect sizes were estimated using phi $(\varphi)$; for all other tests, effect sizes were estimated using correlation $(r$, derived from z-scores; Tomczak and Tomczak, 2014).

\section{Data and software accessibility}

MATLAB analysis code is available on GitHub (https:/github.com/ lexjgomez/Gomez_et_al_2021). Data are available on request.

\section{Results}

\section{Units in S1 and M1 respond to reafferent and exafferent stimulation}

Extracellular unit activity was recorded in S1 and M1 from headfixed rats at P8 and P12 ( $n=8$ pups/age; Fig. $1 A)$. Electrode locations in the forelimb regions of S1 and M1 were confirmed by a neural response to the passive movement of the contralateral forelimb after electrode insertion, and by histology after experiments concluded (Fig. 1B). We recorded a total of $135 \mathrm{~S} 1$ units and 137 M1 units at P8, and 175 S1 units and 231 M1 units at P12. Active sleep accounted for $59.11 \pm 3.4 \%$ of recording time at P8 and $37.7 \pm 4.1 \%$ of recording time at P12. Figure $1 C$ displays representative data for P8 and P12 subjects from periods of active sleep and wake and during the subsequent limb stimulation period. We analyzed neural responses to three types of forelimb events in this first experiment: twitches, wake movements, and passive movements. Each event triggers sensory input that can be characterized along three dimensions: reafferent vs. exafferent, proprioceptive vs. tactile, and active sleep vs. wake (Fig. 1D).

In this first experiment, we characterized differential sensory responses to reafferent and exafferent stimuli at P8 and P12. To do this, we constructed PETHs of z-scored M1 and S1 unit activity triggered on twitches, wake movements, and passive movements (Fig. 2A). In all cases, S1 and M1 neural activity followed the onset of the triggered events, which is indicative of sensory responding. To better characterize S1 and M1 unit activity, we next examined the percentage of all units in both areas that were responsive to reafference from twitches and wake movements, and exafference from passive movements. S1 units were significantly and substantially more responsive than M1 units to exafference at both P8 and P12 (P8: $W_{(7)}=0, p=0.012, r=0.891$; P12: $W_{(7)}=1, p=0.012, r=0.891$; Fig. $\left.2 B\right)$; in contrast, responsiveness to reafference was more variable $\left(H_{(3)}=25.624\right.$, $p<0.001$ ). At P8, both units in S1 and M1 were highly twitch-responsive, although M1 was significantly more responsive than S1 $\left(W_{(7)}=0, p=0.012, r=0.892\right)$; also, at this age, both structures were relatively less responsive to wake movements. At P12, neither structure was very responsive to twitches or wake movements, although S1 was significantly more responsive than $\mathrm{M} 1$ to wake movements $\left(H_{(3)}=14.957, p=0.002 ; W_{(7)}=0, p=0.017\right.$, $r=0.841$ ). These results indicate that $\mathrm{S} 1$ and $\mathrm{M} 1$ process somatosensory reafferent and exafferent input differently at these ages.

\section{Developmental shift in corticocortical signaling between $\mathrm{S1}$ and $\mathrm{M} 1$}

To distinguish between parallel and serial processing of sensory input to S1 and M1, a shift predictor analysis was performed on pairs of units (see Materials and Methods). The resulting corrected histogram indicates the portion of the sensory response that is attributable to interactions between the two units (Fig. 3A; Alloway et al., 1993; Chakrabarti et al., 2008). We computed average corrected histograms for twitches, wake movements, and passive movements within each pup. Individual corrected histograms were separated into those that contributed to a peak ("responsive pairs") and those that did not ("non-responsive pairs"; 
A

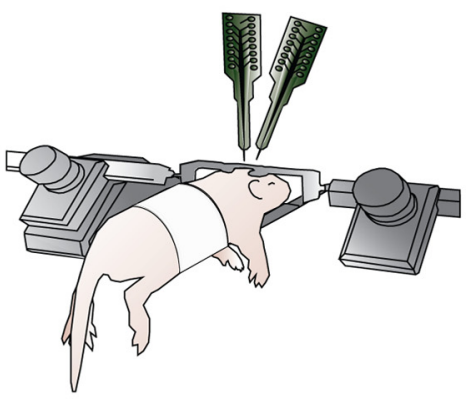

B

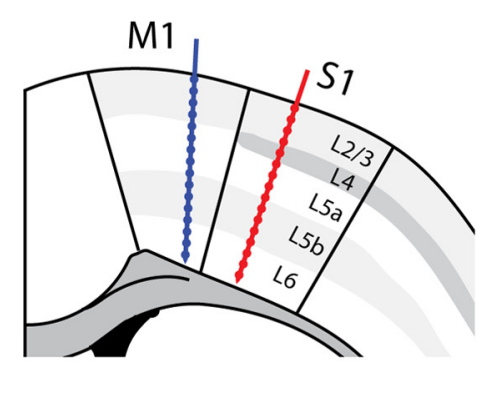

M1

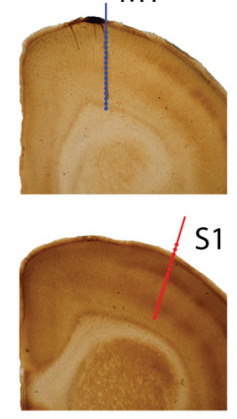

C

P8

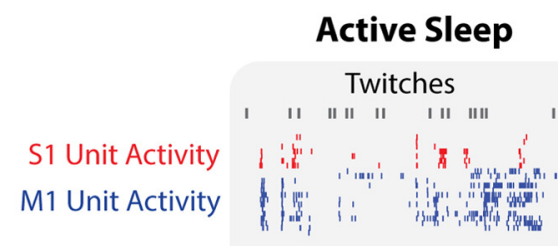

Wake

Wake Movements

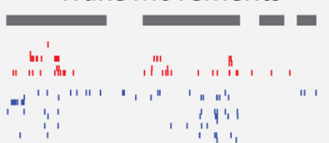

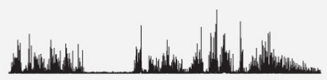

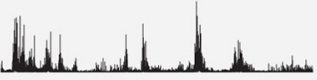

Wake Movements

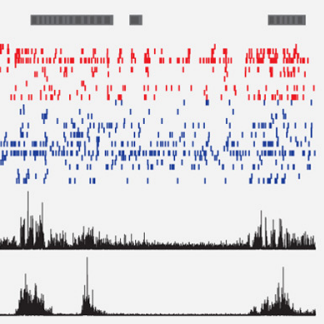

Wake Movements

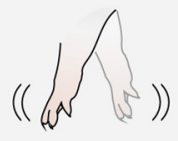

Self

Proprioceptive

Wake

\section{Stimulation Period}

Passive Movements
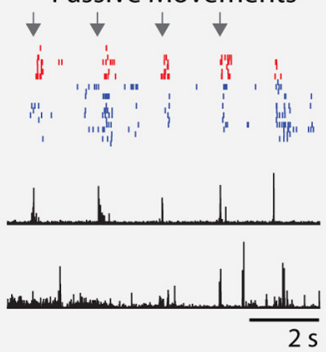

Passive Movements

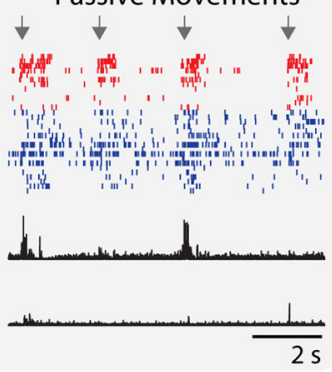

Passive Movements

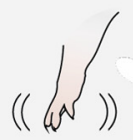

Other

Proprioceptive and Tactile

Active Sleep or Wake

Figure 1. Recording neural activity in S1 and M1 at P8 and P12. $\boldsymbol{A}$, Illustration of a head-fixed rat pup in the stereotaxic apparatus with silicon laminar electrodes inserted in S1 and M1. $\boldsymbol{B}$, left, Illustration of electrode placements in S1 (red) and M1 (blue) in a coronal section of cortex. Right, C0-stained coronal sections, showing reconstruction of electrode placements in M1 (top) and S1 (bottom). C, Representative data from rats at P8 (top) and P12 (bottom) during active sleep and wake as well as periods of passive limb movement. For each record from the top, data are presented as follows: event markers (twitches: ticks; wake movements: solid lines; passive limb movement: arrows), S1 unit activity (red ticks), M1 unit activity (blue ticks), and contralateral forelimb and nuchal EMG records (black traces). D, Depiction of each event type for the first experiment (twitches, wake movements, and passive limb movements) along with the associated source, submodality, and behavioral state.

Fig. $3 B$ ). When a peak was present, the latency of the peak was defined by one of three categories (Fig. 3C).

We first examined the percentage of S1-M1 unit pairs that were responsive to each stimulus type at each age (Fig. 3D). At P8, $98.5 \%$ of all pairs exhibited peaks to twitches and $49.6 \%$ to passive movements; no peaks were observed for wake movements. At P12, 57.7\% of all pairs exhibited peaks to twitches, $34.9 \%$ to wake movements, and $86.3 \%$ to passive movements. Next, focusing on the responsive pairs, we assessed the distribution of peak latencies among the three categories (Fig. 3E). At 


\section{P8}
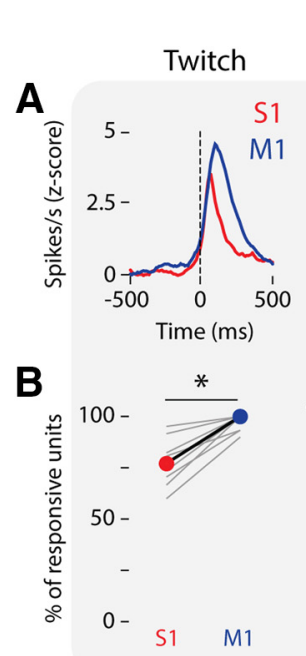

Wake Movement
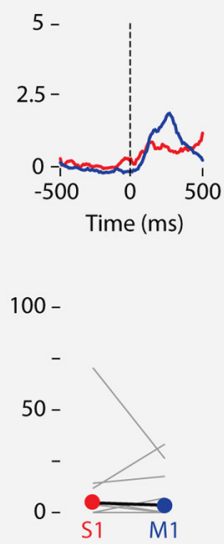

Passive Movement
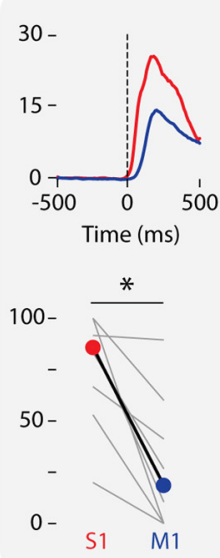

P12

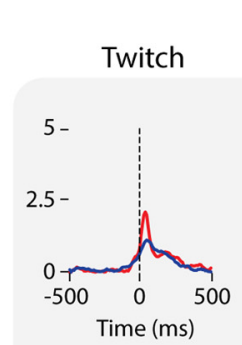

Passive Movement
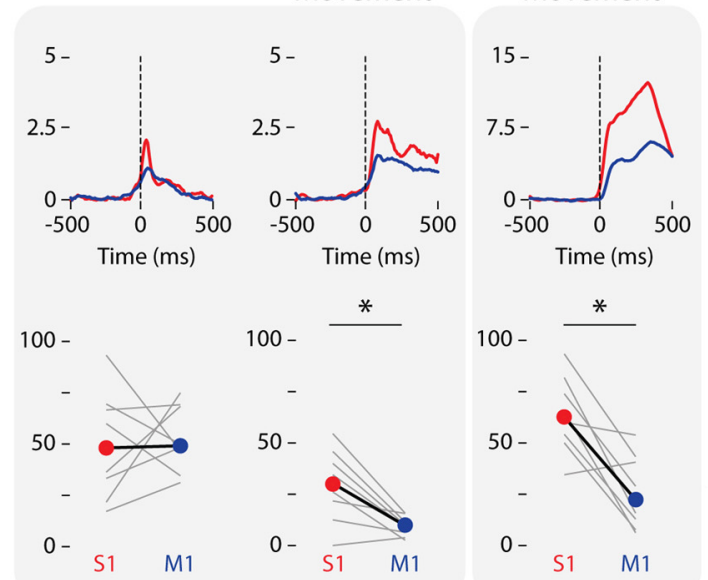
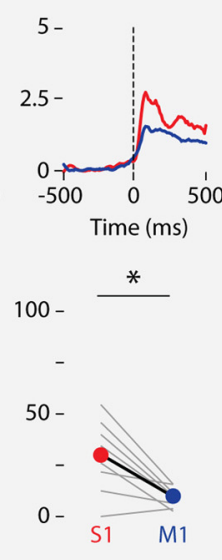

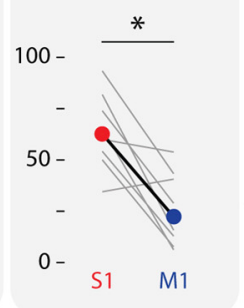

Figure 2. $S 1$ and $M 1$ neural responses to reafference and exafference at $P 8(S 1=135$ units, $M 1=137$ units $)$ and $P 12(S 1=175$ units, $M 1=231$ units). $A, Z$-scored $P E T H s$ of mean firing of units in $\mathrm{S} 1$ (red) and M1 (blue) triggered on a twitch, wake movement, or passive movement. Trigger onset denoted by dashed vertical line at $0 \mathrm{~ms}$. B, Percentage of units in S1 (red) and M1 (blue) that were responsive to sensory events. Red and blue dots denote median values; gray lines denote data for individual animals; $*$ significant difference between $\mathrm{S} 1$ and $\mathrm{M} 1, p<0.025$.

P8, for both twitches and passive movements, the majority of unit pairs exhibited latencies close to zero ( $\pm 4 \mathrm{~ms}$; green shading), indicative of parallel input to S1 and M1. At P12, latencies for twitches and wake movements were evenly divided between those that were close to zero and those that were $>4 \mathrm{~ms}$ (blue shading). The largest shift, however, was for the passive movements group: at P12, $76.8 \%$ of latencies were $>4 \mathrm{~ms}$, indicating a shift in sensory processing from P8 to P12.

To better quantify these latencies differences, we assessed whether the median peak latency for each stimulus type was significantly greater than $4 \mathrm{~ms}$ (Fig. $3 F$ ). At P8, median peak latencies for both twitches and passive limb movements were close to zero (twitch median $=3 \mathrm{~ms}$; passive movement median $=4 \mathrm{~ms}$ ), again indicative of parallel input to S1 and M1. At P12, median latencies for twitches were still close to zero (median $=4 \mathrm{~ms}$ ) as were wake movements (median $=3 \mathrm{~ms}$ ); however, the median latency for passive movements was significantly greater than $4 \mathrm{~ms}$ ( median $\left.=10 \mathrm{~ms} ; W_{(150)}=9284.5, p<0.001, r=0.741\right)$.

In summary, for reafferent stimuli, our results are consistent with those of Dooley and Blumberg (2018) in showing that reafference at P8 and P12 is conveyed to S1 and M1 independently. However, as shown here, exafferent stimuli shift from parallel processing at P8 to serial processing from S1 to M1 at P12. Because the self-generated movements differed from the passive movements in this experiment along two dimensions, the source of the input (self vs. other) and the associated submodality (proprioceptive vs. tactile), we sought to disambiguate those factors in the next experiment.

\section{Responses of units in S1 and M1 to proprioceptive and tactile input}

Self-generated movements entail muscle contraction and consequent activation of proprioceptors (i.e., muscle spindles and Golgi tendon organs; Proske and Gandevia, 2012); because the limbs of the pups in the first experiment dangled freely without touching any surface, we would expect relatively little activation of cutaneous tactile receptors during self-generated movement. In contrast, with passive movement we would expect tactile receptors to be strongly activated on contact with the wooden dowel, followed by activation of proprioceptors as the limb was moved. Accordingly, in this experiment, we aimed to assess the relative contributions of proprioceptors and tactile receptors to the pattern of responses observed in the first experiment. To do this, we contrasted two types of exafferent stimulation: intramuscular stimulation that primarily activates proprioceptors, and cutaneous stimulation that primarily activates tactile receptors. We again recorded from the forelimb regions of S1 and M1 at P8 (134 S1 and $168 \mathrm{M} 1$ units) and P12 (220 S1 and 193 M1 units) rats and analyzed neural responses to each stimulus ( $n=8$ pups/ age).

We first characterized the overall responses to intramuscular and cutaneous stimulation by constructing z-scored PETHs of S1 and M1 activity (Fig. 4A). In general, S1 units were more likely than M1 units to respond to cutaneous stimulation at both ages (Fig. $4 B)$; this difference was significant at P12 $\left(W_{(7)}=0\right.$, $p=0.012, r=0.891)$. In contrast, at both ages the median unit responses to intramuscular stimulation were similar between $S 1$ and M1. We also examined S1 and M1 responses to individual stimulus presentations to understand how frequently S1 and M1 individually responded to each stimulus type (Fig. 4C). At P8 and P12, both S1 and M1 units responded to intramuscular stimulation on a minority of trials. However, for cutaneous stimulation at P8 and at P12, S1 units responded on a significantly higher percentage of trials than M1 units (P8: $U_{(14)}=6$, $p=0.011, r=0.657$; P12: $\left.U_{(15)}=0, p<0.001, r=0.840\right)$.

We observed that the differences between $S 1$ and M1 responses to cutaneous stimulation in this second experiment were similar to those observed for passive movements in the first experiment (Fig. 2A); that is, for both passive movements and cutaneous stimulation, S1 was more responsive than M1. Taken together, these results suggest that the tactile submodality is a more significant driver of differential activity in S1 and M1, rather than the source of the stimulation.

\section{Different pathways to S1 and M1 for processing proprioceptive and tactile inputs}

To determine whether S1 and M1 engage different processing pathways for proprioceptive and tactile input, we again 
A

Mean PETHs

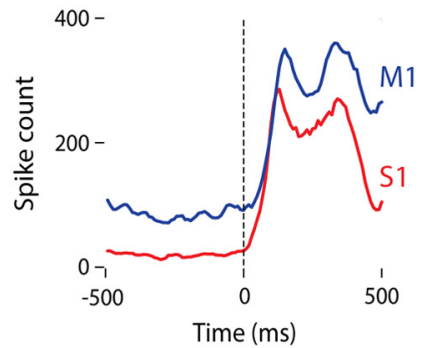

Shift Predictor Analysis

Joint Histogram and Shift Predictor

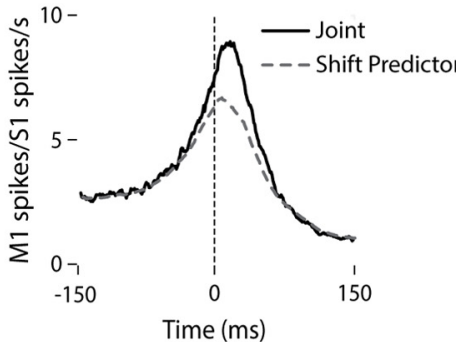

Corrected Histogram

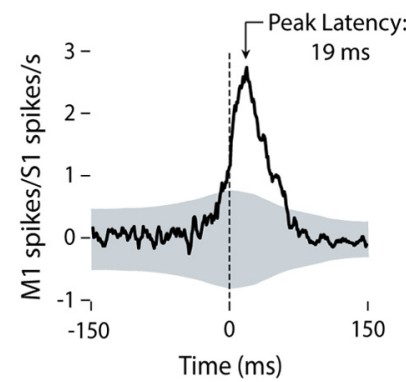

B

\section{Separation of Unit-Unit Pairs}

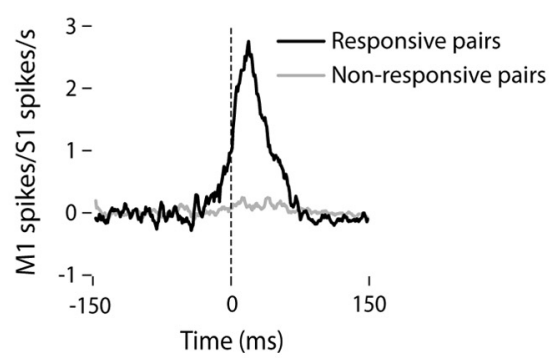

D
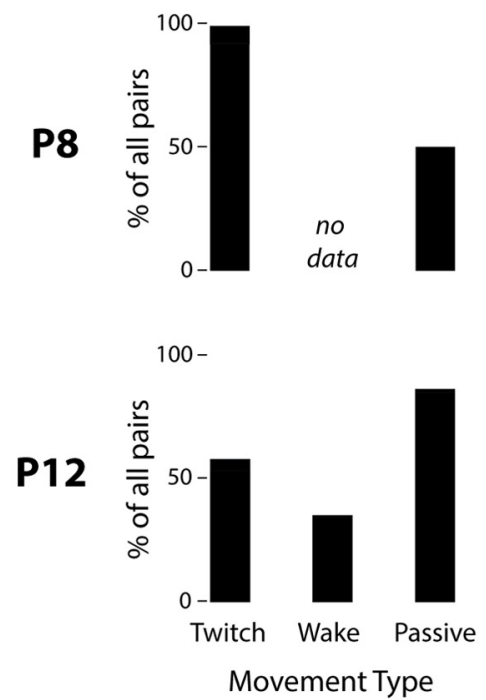

C

\section{Interpretation of Peak Latencies}

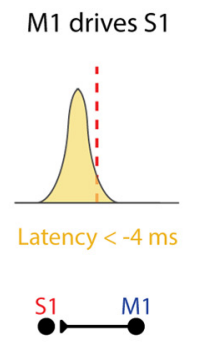

Common input

S1 drives M1
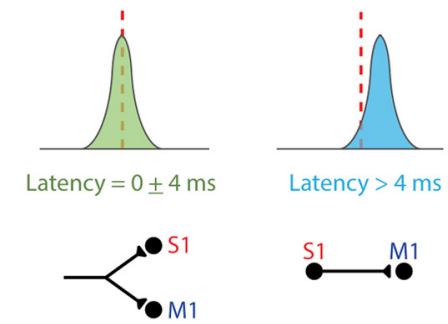

$\mathbf{F}$
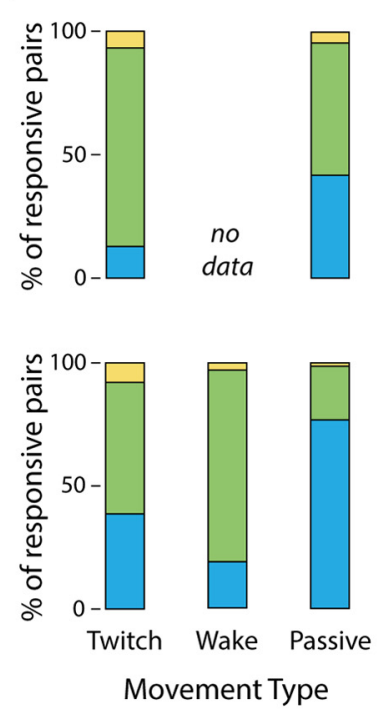
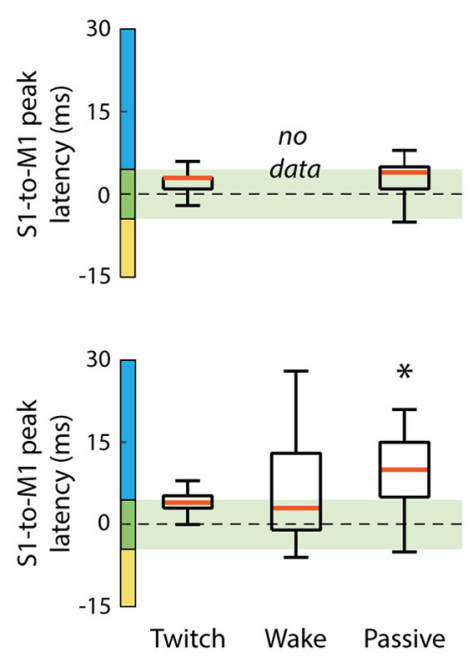

Movement Type

Figure 3. Stimulus-corrected neural activity in relation to reafference and exafference. A, Method for shift predictor analysis. Left, Mean PETHs for S1 (red) and M1 (blue) in response to a sensory event (dashed vertical line). Middle, Joint histogram of M1 activity triggered on $\mathrm{S1}$ activity ("joint," solid line), whose onset is denoted by the dashed vertical line at $0 \mathrm{~ms}$. Also shown is the joint histogram produced by shifting trains of S1 activity within each epoch ("shift predictor," dashed line). Right, Corrected histogram of M1 activity triggered on S1 activity (dashed vertical line); peak latency denoted by arrow. $\boldsymbol{B}$, Corrected histograms of responsive (black line) and non-responsive (gray line) pairs of S1-M1 units. Non-responsive pairs were removed for subsequent analyses. $\boldsymbol{C}$, Cartoons depicting three possible corrected histogram peak latencies and their interpretation: yellow denotes negative peak latency, green denotes peak latency near zero, and blue denotes positive peak latency. $\boldsymbol{D}$, Percentages of all pairs of S1-M1 units at P8 (top) and P12 (bottom) that were responsive to twitches, wake movements, and passive movements. $\boldsymbol{E}$, Stacked plots showing the percentages of responsive pairs of S1-M1 units at P8 (top) and P12 (bottom) that fell into each color-coded category denoted in C. $\boldsymbol{F}$, Boxplots showing the corrected histogram peak latencies for pairs of S1-M1 units at P8 (top) and P12 (bottom) for twitches, wake movements, and passive movements. See $C$ for color coding of $y$-axes; * significant difference from hypothesized median value of $4 \mathrm{~ms}, p<0.001$.

calculated corrected histograms for each S1-M1 unit pair for both stimulation types and at both ages.

The percentage of pairs that exhibited peaks for intramuscular stimulation was higher at P8 (83.6\%) than at P12 (65.0\%). However, for cutaneous stimulation, the percentage of pairs that exhibited peaks doubled from P8 (34.3\%) to P12 (63.2\%; Fig. $5 A$ ). Of these responsive pairs, at $\mathrm{P} 8$, the majority of peak latencies for intramuscular (74.1\%) and cutaneous (60.9\%) stimulation were centered around zero, indicative of parallel input to S1 and M1. At P12, the majority of intramuscular stimulation peak 
P8

\section{Intramuscular Stimulation}
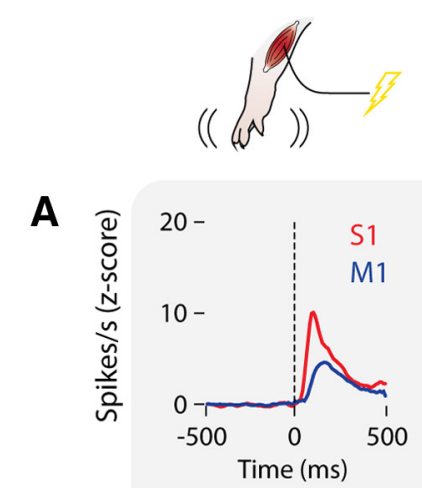

B
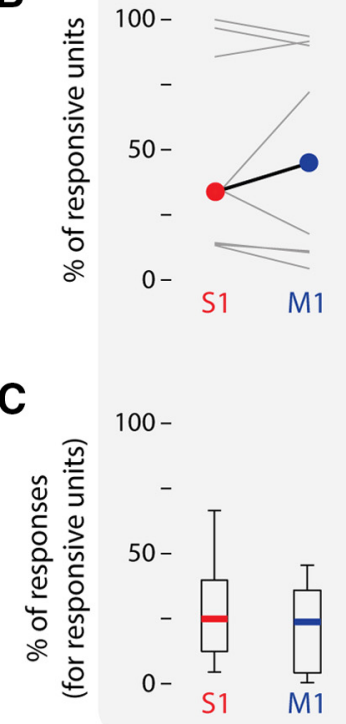

Cutaneous Stimulation
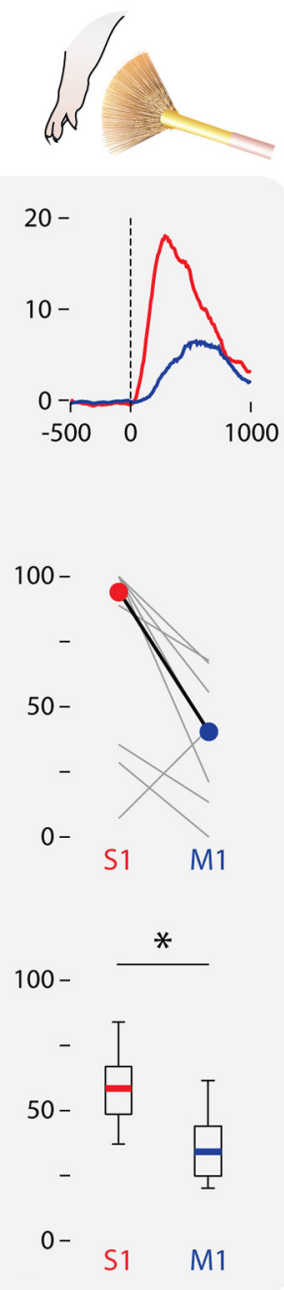

P12
Cutaneous Stimulation

\section{Stimulation}
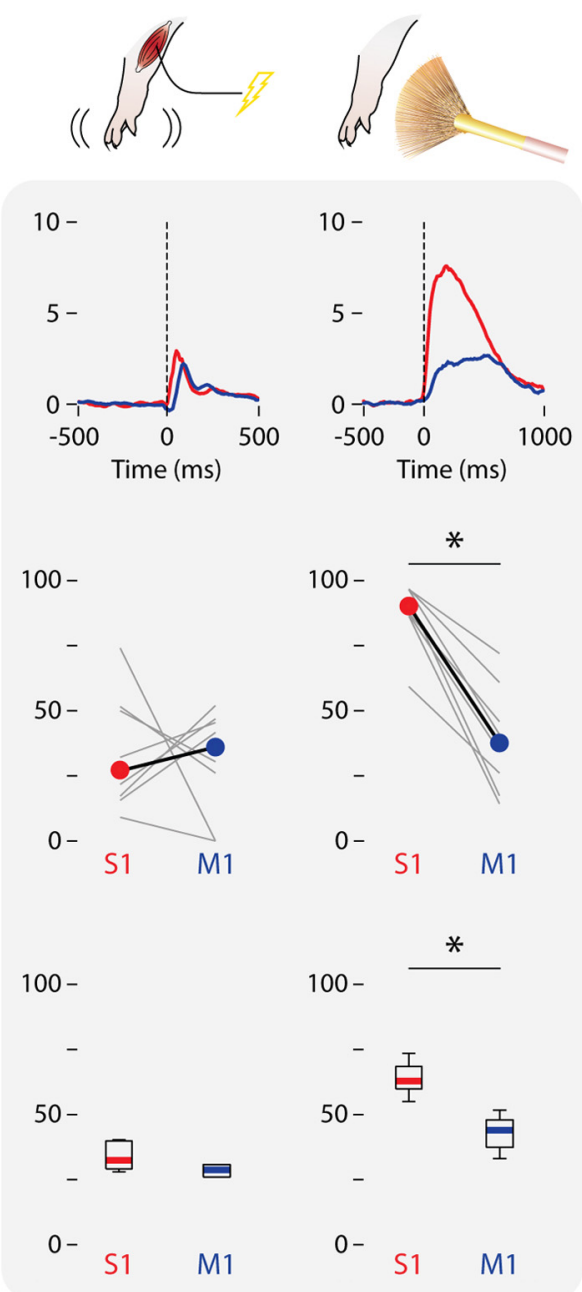

Figure 4. $S 1$ and $M 1$ neural responses to intramuscular and cutaneous stimulation at P8 (S1= 134 units; M1 = 168 units) and P12 (S1=220 units; M1 = 193 units). $\boldsymbol{A}, \mathrm{Z}$-scored PETHs of mean firing of units in S1 (red) and M1 (blue) triggered on intramuscular and cutaneous stimulation (onset denoted by dashed vertical line at 0 ms). $\boldsymbol{B}$, Percentage of units in S1 (red) and M1 (blue) that were responsive to sensory events. Red and blue dots denote median values; gray lines denote data for individual animals; $*$ significant difference between $\mathrm{S} 1$ and $\mathrm{M} 1, p<0.025$. C, Boxplots showing the percentage of stimulations that evoked a response in S1 (red; intramuscular: P8 $=69$ units, P12 = 76 units; cutaneous: P8 = 90 units, P12 = 194 units) and M1 (blue; intramuscular: $\mathrm{P} 8=88$ units, $\mathrm{P} 12=63$ units; cutaneous: $\mathrm{P} 8=66$ units, $\mathrm{P} 12=76$ units); $*$ significant difference between $\mathrm{S} 1$ and $\mathrm{M} 1, p<0.025$.

latencies were still centered around zero (61.5\%), but now $96.4 \%$ of cutaneous stimulation peak latencies were $>4 \mathrm{~ms}$ (Fig. $5 B$ ). There were significant group differences in the proportions of responsive pairs for cutaneous stimulation (P8 vs. P12: $\chi^{2}=97.7$, $p<0.001, \varphi=0.729$ ) and across stimulation types at P12 (intramuscular vs. cutaneous: $\left.\chi^{2}=169.3, p<0.001, \varphi=0.776\right)$. Overall, these results for cutaneous stimulation mirror the shift from parallel to serial processing seen in the first experiment regarding passive movements (Fig. $3 E$ ).

This result is further supported by the individual corrected peak latencies. At P8, the median peak latencies for intramuscular and cutaneous stimulation were both close to zero (intramuscular median = $3 \mathrm{~ms}$; cutaneous median $=2 \mathrm{~ms}$; Fig. $5 \mathrm{C}$ ). At P12, the median latency for intramuscular stimulation remained close to zero (median = $1 \mathrm{~ms}$ ); however, the median latency observed for cutaneous stimulation was $16 \mathrm{~ms}$, significantly greater than our cutoff of $4 \mathrm{~ms}\left(W_{(135)}=9290, p<0.001, r=0.867\right)$, indicating that cutaneous sensory input is processed serially at P12. Now, we have replicated the finding that, at P8, all somatosensory input is processed in parallel, and clarified that by P12 tactile inputs are processed serially from S1 to M1 while proprioceptive inputs continue to be processed in parallel.

However, S1 and M1 do not respond to, and therefore are not coactivated by, every stimulation delivered. To understand how often S1 and M1 were coactivated by the same stimulus, we determined the average percentage of stimulations that elicited responses in both S1 and M1 for those units that contributed to the corrected peak latencies (P8 S1: intramuscular $=112$ units, cutaneous $=46$ units; P8 M1: intramuscular $=145$ units, cutaneous $=58$ units; P12 S1: intramuscular $=143$ units, cutaneous $=139$ units; P12 M1: intramuscular $=127$ units, cutaneous $=121$ units). At P8, we found that, on average, $18.9 \%$ of intramuscular stimulations resulted in coactivation of S1-M1 unit pairs, whereas only $13.4 \%$ of cutaneous stimulations did. At P12, this trend was reversed: only a small 
A
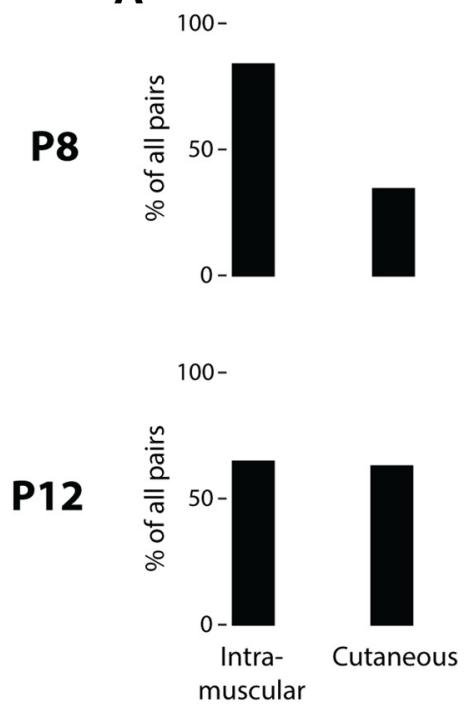

B
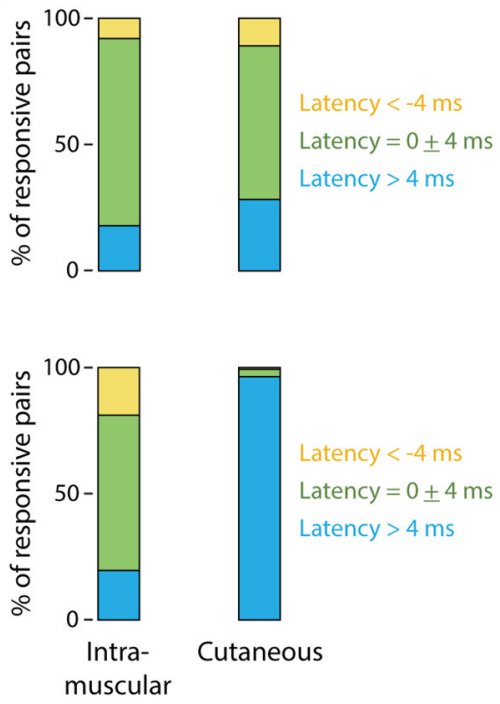

C
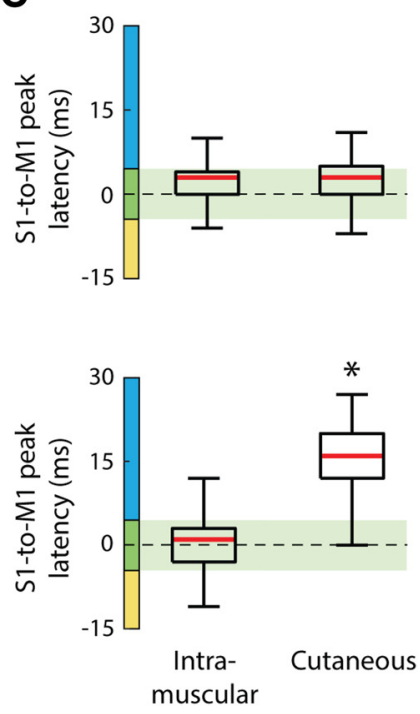

Figure 5. Stimulus-corrected activity in relation to intramuscular and cutaneous stimulation. $\boldsymbol{A}$, Percentages of all pairs of S1-M1 units at P8 (top) and P12 (bottom) that were responsive to intramuscular and cutaneous stimulation. $\boldsymbol{B}$, Stacked plots showing the percentages of responsive pairs of S1-M1 units at P8 (top) and P12 (bottom) that fell into each color-coded category (see Fig. 3C). C, Boxplots showing the peak latencies for the corrected histograms for pairs of S1-M1 units at P8 (top) and P12 (bottom) for intramuscular and cutaneous stimulation. Color coding of $y$-axes the same as in $\boldsymbol{B}$; * significant difference from hypothesized median value of $4 \mathrm{~ms}, p<0.001$.

minority of intramuscular stimulations resulted in coactivation (7.3\%), whereas approximately half of cutaneous stimulations resulted in coactivation (47.8\%). These results indicate that, at P8, proprioceptive inputs produce more coactivation of S1 and M1 than tactile inputs, but that, at P12, tactile inputs produce more coactivation of S1 and M1 than proprioceptive inputs.

\section{State-dependent processing of proprioceptive and tactile inputs}

As shown previously in infant rats, active sleep modulates sensory processing of reafferent input (Tiriac et al., 2014; Dooley and Blumberg, 2018; Mukherjee et al., 2018; Dooley et al., 2020) and may be particularly important for synchronizing developing structures in the sensorimotor system (Del Rio-Bermudez et al., 2020). To determine what role, if any, behavioral state plays in modulating exafferent responses in S1 and M1, we segregated intramuscular and cutaneous stimulations by behavioral state and examined activity in S1 and M1. Not unexpectedly, given the predominance of active sleep at these ages (Seelke and Blumberg, 2010), the majority of intramuscular and cutaneous stimulations at both ages were delivered during active sleep (P8: intramuscular $=69.4 \%$, cutaneous $=$ 65.1\%; P12: intramuscular $=67.2 \%$, cutaneous $=74.0 \%$ ).

PETHs of S1 and M1 unit activity show that neither the magnitude nor the latency of responses differs appreciably between behavioral states for either intramuscular or cutaneous stimulation (Fig. 6A). However, across ages and stimulation types, the corrected histograms of joint S1-M1 activity show that the average peak magnitude was consistently higher for active sleep than for wake at both P8 and P12, although all peaks exceeded the statistical threshold $(p<0.01$; Fig. $6 B)$. To determine whether S1 and M1 fired together more frequently during active sleep compared with wake, we calculated the observed and expected frequencies of S1-M1 unit coactivation in response to intramuscular and cutaneous stimulation (see Materials and Methods). For cutaneous stimulation, S1 and M1 units were coactivated significantly more often during sleep than during wake at both ages $\left(\mathrm{P} 8: W_{(45)}=49, p<0.001, r=0.813 ; \mathrm{P} 12: W_{(138)}=\right.$
2612, $p<0.001, r=0.209)$. In contrast, for intramuscular stimulation, S1-M1 coactivation was not significantly different between sleep and wake.

\section{Discussion}

We have demonstrated that differences in the transmission of sensory input to S1 and M1 depend on submodality and age. At $\mathrm{P} 8, \mathrm{~S} 1$ and $\mathrm{M} 1$ receive tactile and proprioceptive input through parallel pathways. At P12, whereas proprioceptive inputs continue to be processed in parallel, tactile inputs are processed serially from S1 to M1. Finally, at both ages, there is stronger and more frequent coactivation of S1 and M1 units during active sleep than wake.

\section{S1 and M1 develop somatotopy independently}

$\mathrm{S} 1$ and $\mathrm{M} 1$ receive and respond to somatosensory input throughout life. In adults, most sensory input to M1 is thought to depend on the conveyance of signals from S1 (Farkas et al., 1999; Ferezou et al., 2007). Assessments of this dependence in developing rats, however, have yielded inconsistent results; this inconsistency is likely because of methodological differences across studies, such as the use of anesthesia (An et al., 2014) or the focus on reafference or exafference (Dooley and Blumberg, 2018). The present study was designed to systematically assess sensory processing in S1 and M1 to determine when the two structures first develop functional connectivity. Using unanesthetized rats, we found at P8 that $\mathrm{S} 1$ and $\mathrm{M} 1$ receive all of their somatosensory input in parallel (Fig. 7A). As both S1 and M1 require sensory input to develop their functional somatotopies (Keller et al., 1996; Huntley, 1997; Chakrabarty and Martin, 2000; Lendvai et al., 2000; Briner et al., 2010; Young et al., 2012), our finding suggests that S1 and M1 initially develop their somatotopies independently of one another.

The fact that S1 and M1 receive sensory input in parallel at P8 informs our understanding of the anatomic connectivity of each structure at this age. For S1, the pathway that conveys sensory input is well established and conserved across mammalian species (Jones and Friedman, 1982; Rice et al., 1985; Krubitzer and 


\section{Intramuscular Stimulation Separated by Behavioral State}

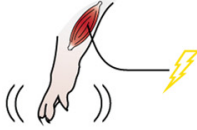

P8

A
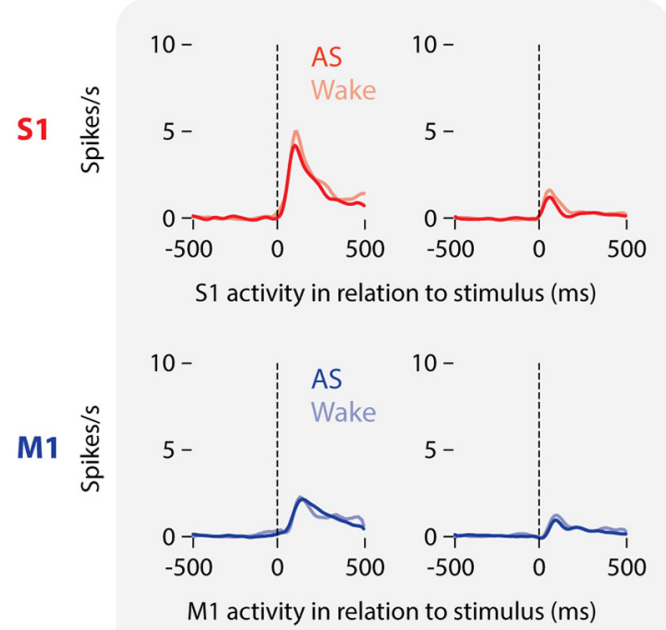

B
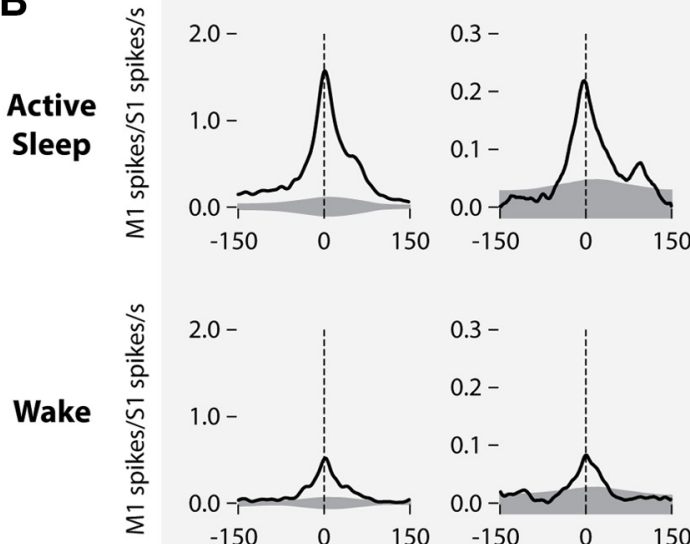
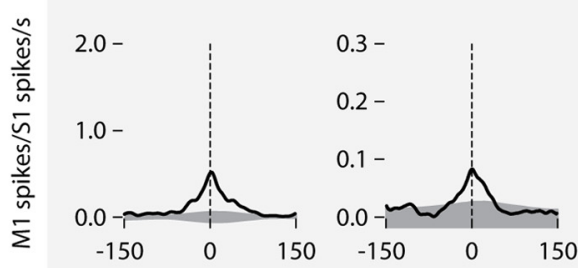

$\mathrm{M} 1$ activity in relation to stimulus-adjacent S1 activity (ms)

\section{Cutaneous Stimulation \\ Separated by Behavioral State}

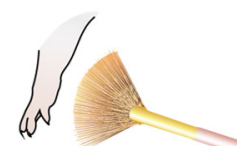

P8

P12
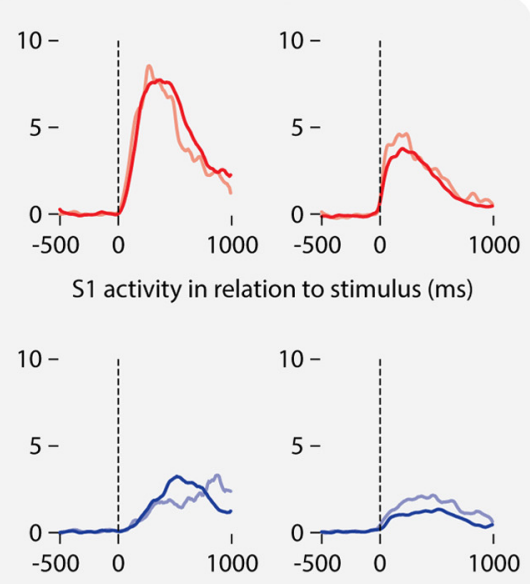

$\mathrm{M} 1$ activity in relation to stimulus (ms)
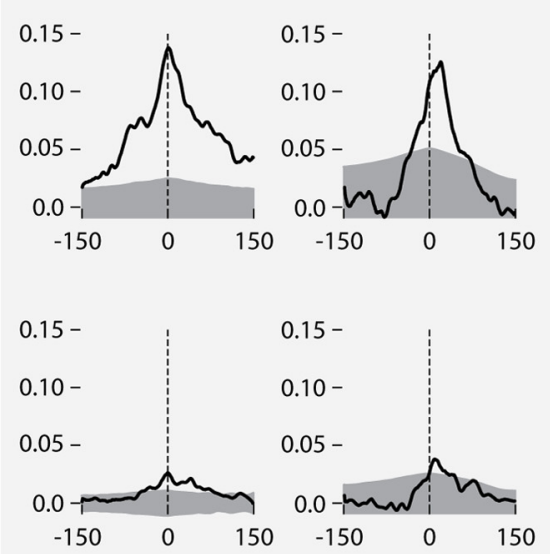

$\mathrm{M} 1$ activity in relation to stimulus-adjacent $\mathrm{S} 1$ activity (ms)

Figure 6. State-dependent modulation of neural activity in $\mathrm{S1}$ and M1 in relation to intramuscular and cutaneous stimulation. $\boldsymbol{A}$, Responses of units in $\mathrm{S1}$ (red, top row) and M1 (blue, bottom row) at P8 and P12 to intramuscular and cutaneous stimulations delivered during active sleep (dark red and blue lines) and wake (light red and blue lines). $\boldsymbol{B}$, Mean corrected histograms of M1 activity triggered on stimulus-adjacent S1 activity (dashed vertical line) intramuscular and cutaneous stimulation delivered during active sleep (top row) and wake (bottom row). Confidence intervals (99\%) are denoted by gray shading.

Kaas, 1987; Erzurumlu and Jhaveri, 1990; Kaas et al., 2008). Briefly, somatosensory input arises from mechanoreceptors in the periphery and is conveyed to several sensory nuclei in the spinal cord and medulla; these nuclei send projections to multiple thalamic nuclei, most notably the ventral posterior (VP) nucleus of the thalamus, that in turn project to S1 (Kaas et al., 2008).

The pathway that conveys sensory input to M1 is less clear. M1 receives direct projections from several thalamic nuclei that themselves receive sensory input from peripheral receptors (Donoghue and Parham, 1983; Asanuma and Mackel, 1989; Hooks et al., 2013, 2015; Mo and Sherman, 2019). Nonetheless, as noted above, M1 has been thought to rely predominantly on S1 for sensory input
(Farkas et al., 1999; Ferezou et al., 2007; Rocco-Donovan et al., 2011; An et al., 2014). The present findings, however, indicate that M1 does not rely on S1 for its somatosensory input, thus suggesting that $\mathrm{M} 1$ receives direct sensory input from the thalamus (Fig. $7 A$ ).

\section{The developmental emergence of functional connectivity between $S 1$ and $M 1$}

In addition to enabling independent somatotopic development, parallel input to S1 and M1 may also facilitate the emergence of corticocortical functional connectivity. Anatomically, corticocortical projections from S1 reach M1 by approximately P8 (Ivy and Killackey, 1982; Kast and Levitt, 2019). However, there appears to be a lag between the arrival of axons and the 
formation of functional connections, perhaps because of a delay in the formation of synapses or the insertion of "silent synapses” (Cohen-Cory, 2002). Silent synapses are prevalent in early development and are so-named because they lack AMPA-receptors and thus do not contribute to action potentials (Isaac et al., 1997; Isaac, 2003; Kerchner and Nicoll, 2008). Importantly, silent synapses contain NMDA-receptors that are activated when the presynaptic and postsynaptic membranes are simultaneously excited; this excitation results in a $\mathrm{Ca}^{2+}$ influx that promotes the insertion of AMPA-receptors, thereby unsilencing the synapse (Liao et al., 2001; Kerchner and Nicoll, 2008). Critically, silent synapses associated with interlaminar projections within S1 (and M1) are unsilenced around P12 in mice (Anastasiades and Butt, 2012). Accordingly, we propose that, early in development, repetitive coactivation of $\mathrm{S} 1$ and M1 from parallel sensory inputs serve to unsilence these synapses around P12, enabling the emergence of functional connectivity.

When functional connectivity between S1 and M1 is expressed at P12, as evidenced by serial processing, it is specific to the tactile submodality. Indeed, at P12, we observed no evidence of parallel processing for tactile input, indicating that the pathway conveying direct tactile input to M1 at P8 is either eliminated or inhibited (Fig. $7 B$ ). In contrast, proprioceptive inputs continue to be processed in parallel at P12. Why? Early corticocortical projections are, at first, exuberant (Innocenti and Price, 2005); over time, exuberant projections are pruned and somatotopically precise connections are strengthened through activity-dependent mechanisms. We propose that the activity provided by parallel proprioceptive input contributes to the developmental alignment of S1 and M1 somatotopic maps. Further, in adults, the presence of short-latency responses in M1 (Asanuma and Mackel, 1989) suggest the persistence of direct proprioceptive inputs to that structure that could help to maintain map alignment.

\section{Units in S1 and M1 are coactivated more often during active sleep than wake}

Active sleep is the most prevalent behavioral state in early development (Roffwarg et al., 1966) and myoclonic twitching is one of that state's most characteristic components (Blumberg et al., 2020). In developing rats, active sleep provides a critical context for the promotion of coherent (i.e., synchronous) neural activity (Del Rio-Bermudez et al., 2017, 2020; Del Rio-Bermudez and

A

B
P8
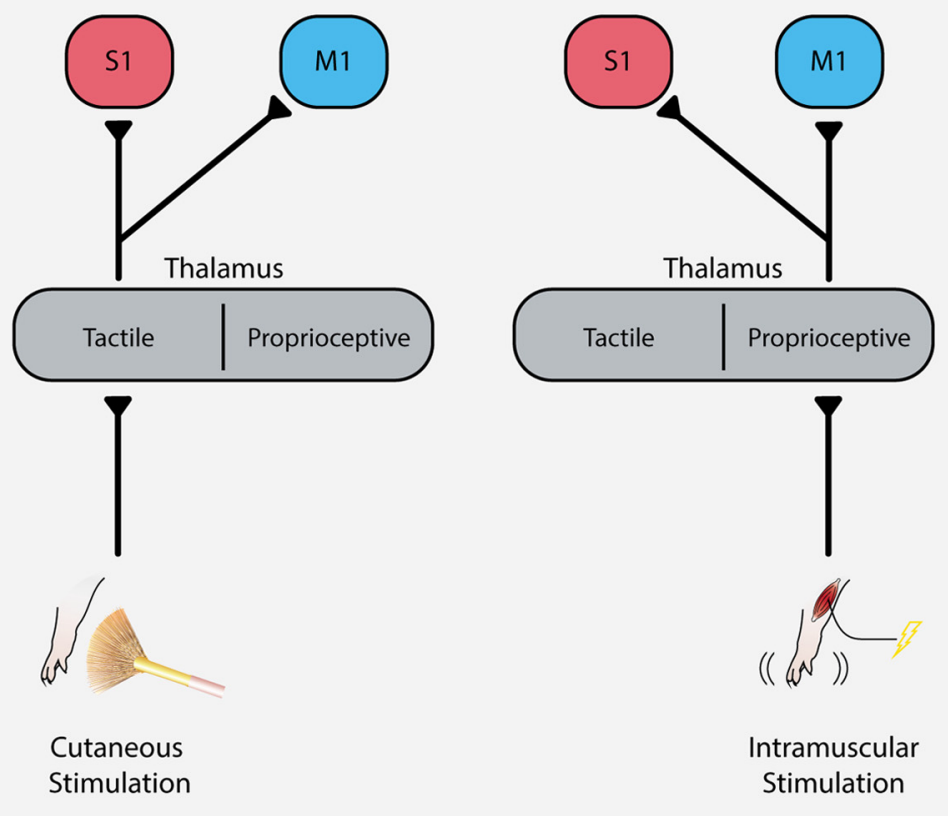

Intramuscular

Stimulation

\section{P12}
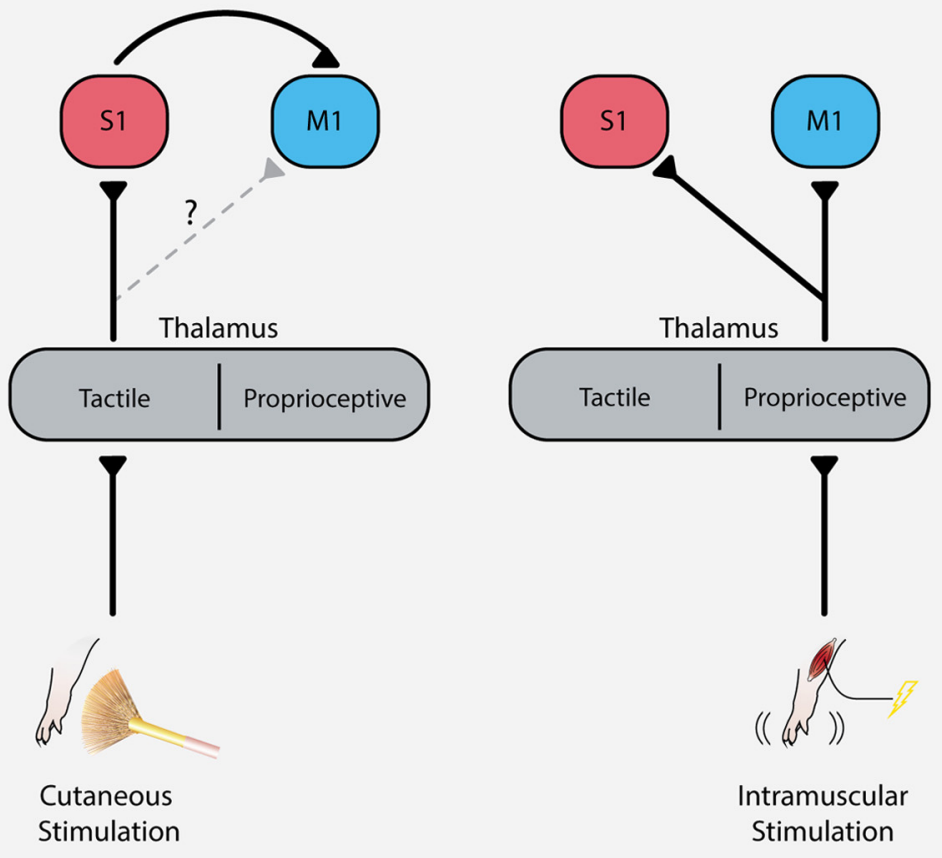

Intramuscular Stimulation

Figure 7. Summary depictions of developmental differences in parallel and serial inputs to $S 1$ and M1. $\boldsymbol{A}$, Parallel tactile (left) and proprioceptive (right) inputs to S1 and M1 at P8. B, Serial tactile (left) and parallel proprioceptive (right) inputs to $\mathrm{S} 1$ and $\mathrm{M} 1$ at P12. Dashed gray line indicates possible suppressed input to M1.

Blumberg, 2018). Also, in early development during active sleep but not wake, reafferent input effectively triggers neural activation in somatosensory structures, including S1 and M1 (Tiriac and Blumberg, 2016; Dooley and Blumberg, 2018; Dooley et al., 2020). Until now, however, the modulatory effects of active sleep on exafferent stimulation had not been systematically assessed. Although we found that active sleep did not influence the 
stimulus-driven responses of S1 and M1 units, it did strongly modulate stimulus-driven coactivation of S1 and M1 units. In short, S1 and M1 fired together more often during active sleep than wake.

We proposed above that coactivation allows S1 and M1 to develop functional connectivity, possibly because of the unsilencing of synapses. Active sleep, by increasing coactivation of S1 and M1, could directly facilitate that development by amplifying and synchronizing activity in sensorimotor structures in ways that cannot be accomplished during other behavioral states. This facilitation may help to explain why active sleep is so prevalent in early development and why self-generated movements (i.e., twitches) are so abundant during that state. Thus, the proprioceptive feedback that arises from twitches and is processed in parallel by S1 and M1 may critically contribute to the development and alignment of their somatotopic maps.

\section{Limitations and future directions}

There are several limitations to this study. First, in our experimental conditions, pups were suspended on a platform that allowed the limbs to dangle freely without contacting any surface. In the ecological context of the nest, however, pups are routinely in contact with their littermates, their mother, and nest material. Thus, the sensory experiences of pups are typically complex and multi-modal (Akhmetshina et al., 2016). As dissimilar as our experimental conditions were from those available in the nest, the results here nonetheless help us to better understand the factors that influence sensory processing under more ecologically relevant conditions.

Second, although we conclude here that S1 and M1 both receive sensory input from thalamus, we cannot yet state with certainty which thalamic nucleus is the source of this input. The most likely source is VP, which projects to S1 (Koralek et al., 1988; Erzurumlu and Jhaveri, 1990) and M1 (Asanuma et al., 1979; Aldes, 1988). However, there are several other nuclei, including the posterior medial nucleus (POm; Donoghue and Parham, 1983; Cicirata et al., 1986; Asanuma and Mackel, 1989) and the ventrolateral nucleus (VL; Cicirata et al., 1986; Yamamoto et al., 1990), that may also convey sensory input to one or both of these cortical areas.

Finally, although we conclude that functional corticocortical connectivity between S1 and M1 emerges by P12, we cannot rule out the contributions of a cortico-thalamo-cortical pathway mediated by POm (Casas-Torremocha et al., 2017, 2019; Mo and Sherman, 2019). Here, at P12, we observed that the median S1to-M1 latency for cutaneous stimulation was $16 \mathrm{~ms}$, longer than latencies reported in adult rats (Farkas et al., 1999; Chakrabarti et al., 2008). Such long latencies could be because of axonal conduction delays (Salami et al., 2003) or reliance on trans-thalamic pathways via POm. Thus, one important next step in achieving a more comprehensive understanding of sensory development in S1 and M1 is to better understand the diversity of their interconnections with thalamic nuclei (Sherman, 2016; Halassa and Sherman, 2019).

\section{References}

Akhmetshina D, Nasretdinov A, Zakharov A, Valeeva G, Khazipov R (2016) The nature of the sensory input to the neonatal rat barrel cortex. J Neurosci 36:9922-9932.

Aldes LD (1988) Thalamic connectivity of rat somatic motor cortex. Brain Res Bull 20:333-348.

Alloway KD, Johnson MJ, Wallace MB (1993) Thalamocortical interactions in the somatosensory system: interpretations of latency and cross-correlation analyses. J Neurophysiol 70:892-908.
An S, Kilb W, Luhmann HJ (2014) Sensory-evoked and spontaneous gamma and spindle bursts in neonatal rat motor cortex. J Neurosci 34:1087010883 .

Anastasiades PG, Butt SJB (2012) A role for silent synapses in the development of the pathway from layer $2 / 3$ to 5 pyramidal cells in the neocortex. J Neurosci 32:13085-13099.

Asanuma H, Mackel R (1989) Direct and indirect sensory input pathways to the motor cortex; its structure and function in relation to learning of motor skills. Jpn J Physiol 39:1-19.

Asanuma H, Larsen KD, Zarzecki P (1979) Peripheral input pathways projecting to the motor cortex in the cat. Brain Res 172:197-208.

Blumberg MS, Sokoloff G, Tiriac A, Del Rio-Bermudez C (2015) A valuable and promising method for recording brain activity in behaving newborn rodents. Dev Psychobiol 57:506-517.

Blumberg MS, Lesku JA, Libourel PA, Schmidt MH, Rattenborg NC (2020) What is REM sleep? Curr Biol 30:R38-R49.

Briner A, De Roo M, Dayer A, Muller D, Kiss JZ, Vutskits L (2010) Bilateral whisker trimming during early postnatal life impairs dendritic spine development in the mouse somatosensory barrel cortex. J Comp Neurol 518:1711-1723.

Casas-Torremocha D, Clascá F, Núñez Á (2017) Posterior thalamic nucleus modulation of tactile stimuli processing in rat motor and primary somatosensory cortices. Front Neural Circuits 11:69.

Casas-Torremocha D, Porrero C, Rodriguez-Moreno J, García-Amado M, Lübke JHR, Núñez Á, Clascá F (2019) Posterior thalamic nucleus axon terminals have different structure and functional impact in the motor and somatosensory vibrissal cortices. Brain Struct Funct 224:1627-1645.

Chakrabarty S, Martin JH (2000) Postnatal development of the motor representation in primary motor cortex. J Neurophysiol 84:25822594.

Chakrabarty S, Martin JH (2005) Motor but not sensory representation in motor cortex depends on postsynaptic activity during development and in maturity. J Neurophysiol 94:3192-3198.

Chakrabarti S, Zhang M, Alloway KD (2008) MI neuronal responses to peripheral whisker stimulation: relationship to neuronal activity in SI barrels and septa. J Neurophysiol 100:50-63.

Cicirata F, Angaut P, Cioni M, Serapide MF, Papale A (1986) Functional organization of thalamic projections to the motor cortex. An anatomical and electrophysiological study in the rat. Neuroscience 19:81-99.

Cohen-Cory S (2002) The developing synapse: construction and modulation of synaptic structures and circuits. Science 298:770-776.

Crapse TB, Sommer MA (2008) Corollary discharge across the animal kingdom. Nat Rev Neurosci 9:587-600.

Curry IJJ, Heim LM (1966) Brain myelination after neonatal administration of oestradiol. Nature 209:915-916.

Del Rio-Bermudez C, Blumberg MS (2018) Active sleep promotes functional connectivity in developing sensorimotor networks. BioEssays 40:1700234.

Del Rio-Bermudez C, Kim J, Sokoloff G, Blumberg MS (2017) Theta oscillations during active sleep synchronize the developing rubro-hippocampal sensorimotor network. Curr Biol 27:1413-1424.

Del Rio-Bermudez C, Kim J, Sokoloff G, Blumberg MS (2020) Active sleep promotes coherent oscillatory activity in the cortico-hippocampal system of infant rats. Cereb Cortex 30:2070-2082.

Donoghue JP, Parham C (1983) Afferent connections of the lateral agranular field of the rat motor cortex. J Comp Neurol 217:390-404.

Dooley JC, Blumberg MS (2018) Developmental "awakening" of primary motor cortex to the sensory consequences of movement. Elife 7:e41841.

Dooley JC, Glanz RM, Sokoloff G, Blumberg MS (2020) Self-generated whisker movements drive state-dependent sensory input to developing barrel cortex. Curr Biol 30:2404-2410.

Ebbesen CL, Insanally MN, Kopec CD, Murakami M, Saiki A, Erlich JC (2018) More than just a "motor": recent surprises from the frontal cortex. J Neurosci 38:9402-9413.

Farkas T, Kis Z, Toldi J, Wolff JR (1999) Activation of the primary motor cortex by somatosensory stimulation in adult rats is mediated mainly by associational connections from the somatosensory cortex. Neuroscience 90:353-361.

Ferezou I, Haiss F, Gentet LJ, Aronoff R, Weber B, Petersen CCH (2007) Spatiotemporal dynamics of cortical sensorimotor integration in behaving mice. Neuron 56:907-923. 
Goldring S, Aras E, Weber PC (1970) Comparative study of sensory input to motor cortex in animals and man. Electroencephalogr Clin Neurophysiol 29:537-550.

Halassa MM, Sherman SM (2019) Thalamocortical circuit motifs: a general framework. Neuron 103:762-770.

Halley AC, Baldwin MKL, Cooke DF, Englund M, Krubitzer L (2020) Distributed motor control of limb movements in rat motor and somatosensory cortex: the sensorimotor amalgam revisited. Cereb Cortex 30:6296-6312.

Hatsopoulos NG, Suminski AJ (2011) Sensing with the motor cortex. Neuron 72:477-487.

Herman D, Kang R, MacGillis M, Zarzecki P (1985) Responses of cat motor cortex neurons to cortico-cortical and somatosensory inputs. Exp Brain Res 57:598-604.

Holson RR, Pearce B (1992) Principles and pitfalls in the analysis of prenatal treatment effects in multiparous species. Neurotoxicol Teratol 14:221228 .

Horne MK, Tracey DJ (1979) The afferents and projections of the ventroposterolateral thalamus in the monkey. Exp Brain Res 36:129-141.

Hooks BM, Hires SA, Zhang YX, Huber D, Petreanu L, Svoboda K, Shepherd GMG (2011) Laminar analysis of excitatory local circuits in vibrissal motor and sensory cortical areas. PLoS Biol 9:e1000572.

Hooks BM, Mao T, Gutnisky DA, Yamawaki N, Svoboda K, Shepherd GMG (2013) Organization of cortical and thalamic input to pyramidal neurons in mouse motor cortex. J Neurosci 33:748-760.

Hooks BM, Lin JY, Guo C, Svoboda K (2015) Dual-channel circuit mapping reveals sensorimotor convergence in the primary motor cortex. J Neurosci 35:4418-4426.

Huntley GW (1997) Differential effects of abnormal tactile experience on shaping representation patterns in developing and adult motor cortex. J Neurosci 17:9220-9232.

Innocenti GM, Price DJ (2005) Exuberance in the development of cortical networks. Nat Rev Neurosci 6:955-965.

Isaac JTR (2003) Postsynaptic silent synapses: evidence and mechanisms. Neuropharmacology 45:450-460.

Isaac JTR, Crair MC, Nicoll RA, Malenka RC (1997) Silent synapses during development of thalamocortical inputs. Neuron 18:269-280.

Ivy GO, Killackey HP (1982) Ontogenetic changes in the projections of neocortical neurons. J Neurosci 2:735-743.

Jones EG, Friedman DP (1982) Projection pattern of functional components of thalamic ventrobasal complex on monkey somatosensory cortex. J Neurophysiol 48:521-544.

Kaas JH, Qi H, Burish M, Omar G, Onifer SM, Massey JM (2008) Cortical and subcortical plasticity in the brains of humans, primates, and rats after damage to sensory afferents in the dorsal columns of the spinal cord. Exp Neurol 209:407-416.

Kast RJ, Levitt P (2019) Precision in the development of neocortical architecture: from progenitors to cortical networks. Prog Neurobiol 175:77-95.

Keller A, Weintraub ND, Miyashita E (1996) Tactile experience determines the organization of movement representations in rat motor cortex. Neuroreport 7:2373-2378.

Kerchner GA, Nicoll RA (2008) Silent synapses and the emergence of a postsynaptic mechanism for LTP. Nat Rev Neurosci 9:813-825.

Koralek KA, Jensen KF, Killackey HP (1988) Evidence for two complementary patterns of thalamic input to the rat somatosensory cortex. Brain Res 463:346-351.

Krubitzer LA, Kaas JH (1987) Thalamic connections of three representations of the body surface in somatosensory cortex of gray squirrels. J Comp Neurol 265:549-580.

Lemon RN, van der Burg J (1979) Short-latency peripheral inputs to thalamic neurones projecting to the motor cortex in the monkey. Exp Brain Res 36:445-462.

Lendvai B, Stern EA, Chen B, Svoboda K (2000) Experience-dependent plasticity of dendritic spines in the developing rat barrel cortex in vivo. Nature 404:876-881.

Liao D, Scannevin RH, Huganir R (2001) Activation of silent synapses by rapid activity-dependent synaptic recruitment of AMPA receptors. J Neurosci 21:6008-6017.

Mao T, Kusefoglu D, Hooks BM, Huber D, Petreanu L, Svoboda K (2011) Long-range neuronal circuits underlying the interaction between sensory and motor cortex. Neuron 72:111-123.
Marques-Smith A, Lyngholm D, Kaufmann AK, Stacey JA, HoerderSuabedissen A, Becker EBE, Wilson MC, Molnár Z, Butt SJB (2016) A transient translaminar GABAergic interneuron circuit connects thalamocortical recipient layers in neonatal somatosensory cortex. Neuron 89:536-549.

Martin JH (2005) The corticospinal system: from development to motor control. Neuroscientist 11:161-173.

Mathis MW, Mathis A, Uchida N (2017) Somatosensory cortex plays an essential role in forelimb motor adaptation in mice. Neuron 93:14931503.e6.

Matyas F, Sreenivasan V, Marbach F, Wacongne C, Barsy B, Mateo C, Aronoff R, Petersen CCH (2010) Motor control by sensory cortex. Science 330:1240-1244.

McVea DA, Mohajerani MH, Murphy TH (2012) Voltage-sensitive dye imaging reveals dynamic spatiotemporal properties of cortical activity after spontaneous muscle twitches in the newborn rat. J Neurosci 32:10982-10994.

Mengler L, Khmelinskii A, Diedenhofen M, Po C, Staring M, Lelieveldt BPF, Hoehn M (2014) Brain maturation of the adolescent rat cortex and striatum: changes in volume and myelination. Neuroimage 84:35-44.

Mo C, Sherman SM (2019) A sensorimotor pathway via higher-order thalamus. J Neurosci 39:692-704.

Mukherjee D, Sokoloff G, Blumberg MS (2018) Corollary discharge in precerebellar nuclei of sleeping infant rats. Elife 7:e38213.

Pachitariu M, Steinmetz N, Kadir S, Carandini M, Harris K (2016) Fast and accurate spike sorting of high-channel count probes with KiloSort. Adv Neural Inf Process Syst :4455-4463.

Pavlides C, Miyashita E, Asanuma H (1993) Projection from the sensory to the motor cortex is important in learning motor skills in the monkey. J Neurophysiol 70:733-741.

Petrof I, Viaene AN, Sherman SM (2015) Properties of the primary somatosensory cortex projection to the primary motor cortex in the mouse. J Neurophysiol 113:2400-2407.

Proske U, Gandevia SC (2012) The proprioceptive senses: their roles in signaling body shape, body position and movement, and muscle force. Physiol Rev 92:1651-1697.

Rice FL, Gomez C, Barstow C, Burnet A, Sands P (1985) A comparative analysis of the development of the primary somatosensory cortex: interspecies similarities during barrel and laminar development. J Comp Neurol 236:477-495.

Rocco-Donovan M, Ramos RL, Giraldo S, Brumberg JC (2011) Characteristics of synaptic connections between rodent primary somatosensory and motor cortices. Somatosens Mot Res 28:63-72.

Roffwarg HP, Muzio JN, Dement WC (1966) Ontogenetic development of the human sleep-dream cycle. Science 152:604-619.

Rosenkranz K, Rothwell JC (2012) Modulation of proprioceptive integration in the motor cortex shapes human motor learning. J Neurosci 32:90009006.

Rossant C, Harris KD (2013) Hardware-accelerated interactive data visualization for neuroscience in Python. Front Neuroinform 7:36.

Erzurumlu RS, Jhaveri S (1990) Thalamic axons confer a blueprint of the sensory periphery onto the developing rat somatosensory cortex. Dev Brain Res 56:229-234.

Sakamoto T, Arissian K, Asanuma H (1989) Functional role of the sensory cortex in learning motor skills in cats. Brain Res 503:258-264.

Salami M, Itami C, Tsumoto T, Kimura F (2003) Change of conduction velocity by regional myelination yields constant latency irrespective of distance between thalamus and cortex. Proc Natl Acad Sci USA 100:61746179.

Sasaki K, Gemba H (1984) Compensatory motor function of the somatosensory cortex for the motor cortex temporarily impaired by cooling in the monkey. Exp Brain Res 55:60-68.

Seelke AMH, Blumberg MS (2008) The microstructure of active and quiet sleep as cortical delta activity emerges in infant rats. Sleep 31:691-699.

Seelke AMH, Blumberg MS (2010) Developmental appearance and disappearance of cortical events and oscillations in infant rats. Brain Res 1324:34-42.

Sherman SM (2016) Thalamus plays a central role in ongoing cortical functioning. Nat Neurosci 19:533-541.

Singleton AC, Brown AR, Teskey GC (2021) Development and plasticity of complex movement representations. J Neurophysiol, in press. 
Tiriac A, Blumberg MS (2016) Gating of reafference in the external cuneate nucleus during self-generated movements in wake but not sleep. Elife 5: e18749.

Tiriac A, Rio-Bermudez CD, Blumberg MS (2014) Self-generated movements with "unexpected" sensory consequences. Curr Biol 24:2136-2141.

Tomczak M, Tomczak E (2014) The need to report effect size estimates revisited. An overview of some recommended measures of effect size. Trends Sport Sci 1:19-25.

Tracey DJ, Asanuma C, Jones EG, Porter R (1980) Thalamic relay to motor cortex: afferent pathways from brain stem, cerebellum, and spinal cord in monkeys. J Neurophysiol 44:532-554.

Tukey JW (1977) Exploratory data analysis. Boston: Addison-Wesley Publishing Company.
Umeda T, Isa T, Nishimura Y (2019) The somatosensory cortex receives information about motor output. Sci Adv 5:eaaw5388.

Vidoni ED, Acerra NE, Dao E, Meehan SK, Boyd LA (2010) Role of the primary somatosensory cortex in motor learning: an rTMS study. Neurobiol Learn Mem 93:532-539.

Yamamoto T, Kishimoto Y, Yoshikawa H, Oka H (1990) Cortical laminar distribution of rat thalamic ventrolateral fibers demonstrated by the PHA-L anterograde labeling method. Neurosci Res 9:148-154.

Young NA, Vuong J, Teskey GC (2012) Development of motor maps in rats and their modulation by experience. J Neurophysiol 108:1309-1317.

Zagha E, Casale AE, Sachdev RNS, McGinley MJ, McCormick DA (2013) Motor cortex feedback influences sensory processing by modulating network state. Neuron 79:567-578. 